\title{
GRAZING BIFURCATIONS IN PERIODIC HYBRID SYSTEMS
}

\author{
Vaibhav Donde \\ Dept. of Elec. and Comp. Engineering \\ University of Illinois at UC \\ Urbana, IL 61801 \\ donde@ece.uiuc.edu
}

\begin{abstract}
Grazing bifurcations occur when a small parameter variation induces a change in the event sequence of a hybrid system, i.e., a system where continuous dynamics and discrete events strongly interact. At such a bifurcation, the system trajectory makes tangential contact with (grazes) an event triggering hypersurface. This bounding case separates regions of (generally) quite different dynamic behaviour. The paper formulates the conditions governing grazing bifurcation points, and extends those conditions to limit cycles. A shooting method is used to solve for bifurcating limit cycles. The approach is applicable for general nonlinear hybrid systems.
\end{abstract}

\section{INTRODUCTION}

Grazing bifurcations [1, 2, 3] refer to situations where a small change in parameter value results in a change in event triggering, with a subsequent divergence of dynamic behaviour. Figure 1 provides an illustration. For a certain value of parameter $\lambda^{+}$, the system trajectory encounters an event triggering hypersurface at a point $x^{+}$. The event occurs, and the trajectory continues accordingly. However for a small change in parameter value to $\lambda^{-}$, the trajectory misses (at least locally) the triggering hypersurface, and subsequently exhibits a completely different form of response. At a parameter value $\lambda_{g}$, lying between $\lambda^{+}$and $\lambda^{-}$, the continuous trajectory tangentially encounters the triggering hypersurface. This bounding case describes a grazing bifurcation, with $\lambda_{g}$ referring to the critical value of the bifurcation parameter.

Hybrid systems are typified by strong coupling between continuous dynamics and discrete events. For such systems, event triggering generally has a significant influence over subsequent system behaviour. Therefore identifying grazing bifurcations, where small parameter changes alter the event triggering pattern, is particularly important. However the non-smooth nature of trajectories has traditionally complicated this process.

Previous investigations of grazing bifurcations (and closely related border collision bifurcations) have focused largely on classifying the (local) consequences of bifurcations through analysis of eigenvalue behaviour. Efforts have been directed primarily towards periodic systems. Computation of actual bifurcation points has generally received little attention. With numerical packages such as AUTO [4] unsuited to non-smooth systems, ad hoc approaches have prevailed. Computation of bifurcation points for non-periodic systems was recently addressed in [5]. This current

Research supported by the National Science Foundation through grant ECS-0332777.

\author{
Ian A. Hiskens
}

\author{
Dept. of Elec. and Comp. Engineering \\ University of Wisconsin-Madison \\ Madison, WI 53706 \\ hiskens@engr.wisc.edu
}

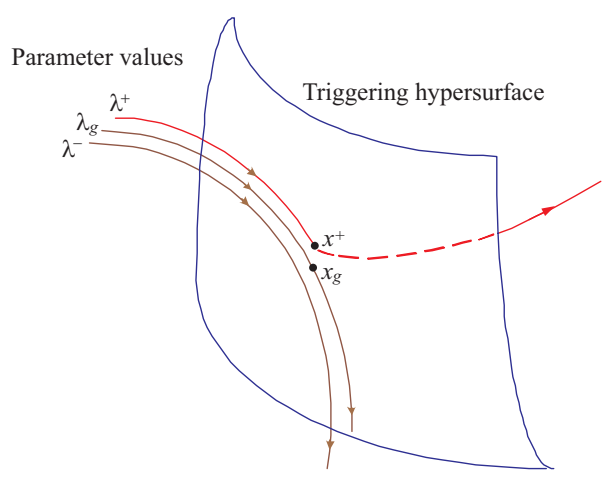

Fig. 1. Grazing bifurcation.

paper establishes a shooting method for finding bifurcating limit cycles of nonlinear hybrid systems.

\section{PROBLEM FORMULATION}

\subsection{Hybrid system model}

It is shown in $[6,7]$ that the continuous/discrete dynamic behaviour of hybrid systems can be captured by a model that consists of a set of differential-algebraic equations, adapted to incorporate impulsive (state reset) action and switching of the algebraic equations. This

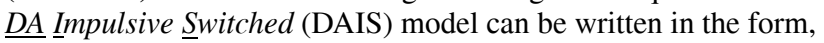

$$
\begin{aligned}
\dot{x} & =f(x, y) \\
x^{+} & =h_{j}\left(x^{-}, y^{-}\right) \quad \text { when } y_{r, j}=0 \\
0 & =g(x, y) \equiv g^{(0)}(x, y)+\sum_{i=1}^{s} g^{(i)}(x, y)
\end{aligned}
$$

where

$$
g^{(i)}(x, y)=\left\{\begin{array}{ll}
g^{(i-)}(x, y) & y_{s, i}<0 \\
g^{(i+)}(x, y) & y_{s, i}>0
\end{array} \quad i=1, \ldots, s\right.
$$

and

- $x \in \mathbb{R}^{n}$ are dynamic states, and $y \in \mathbb{R}^{m}$ are algebraic states;

- $x^{+}$denotes the value of $x$ just after the reset event, while $x^{-}$and $y^{-}$refer to the values of $x$ and $y$ just prior to the event.

- $y_{r}, y_{s}$ are selected elements of $y$ that trigger state reset (impulsive) and algebraic switching events respectively; $y_{r}$ and $y_{s}$ may share common elements. 
A compact development of the equations describing grazing bifurcations results from incorporating system parameters $\lambda \in \mathbb{R}^{\ell}$ into the dynamic states $x$. This is achieved by introducing trivial differential equations

$$
\dot{\lambda}=0
$$

into (1), and results in the natural partitioning

$$
x=\left[\frac{x}{\lambda}\right], \quad f=\left[\frac{f}{0}\right], \quad h_{j}=\left[\begin{array}{c}
\underline{h}_{j} \\
\lambda
\end{array}\right]
$$

where $\underline{x}$ are the true dynamic states and $\lambda$ are parameters.

Away from events, system dynamics evolve smoothly according to the familiar differential-algebraic model

$$
\begin{aligned}
\dot{x} & =f(x, y) \\
0 & =g(x, y)
\end{aligned}
$$

where $g$ is composed of $g^{(0)}$ together with appropriate choices of $g^{(i-)}$ or $g^{(i+)}$, depending on the signs of the corresponding elements of $y_{s}$. At switching events (4), some component equations of $g$ change. To satisfy the new $g=0$ constraints, algebraic variables $y$ may undergo a step change. Impulse events (2) force a discrete change in elements of $x$. Algebraic variables may again step to ensure $g=0$ is always satisfied.

The flows of $x$ and $y$ are defined as

$$
\begin{aligned}
& x(t)=\phi_{1}\left(x_{0}, t\right) \\
& y(t)=\phi_{2}\left(x_{0}, t\right)
\end{aligned}
$$

where $x(t)$ and $y(t)$ satisfy (1)-(4), along with initial conditions,

$$
\phi_{1}\left(x_{0}, t_{0}\right)=x_{0} \quad g\left(x_{0}, \phi_{y}\left(x_{0}, t_{0}\right)\right)=0 .
$$

\subsection{Grazing bifurcations}

A grazing bifurcation is characterised by a trajectory (flow) of the system touching a triggering hypersurface tangentially. Let the target hypersurface be described by

$$
b(x, y)=0
$$

where $b: \mathbb{R}^{n+m} \rightarrow \mathbb{R}$. Vectors that are normal to $b$ are given by $\nabla b=\left[\begin{array}{ll}\frac{\partial b}{\partial x} & \frac{\partial b}{\partial y}\end{array}\right]^{T} \equiv\left[\begin{array}{ll}b_{x} & b_{y}\end{array}\right]^{T}$, and the tangent hyperplane is spanned by vectors $\left[\begin{array}{ll}u^{T} & v^{T}\end{array}\right]^{T}$ that satisfy

$$
\left[\begin{array}{ll}
b_{x} & b_{y}
\end{array}\right]\left[\begin{array}{l}
u \\
v
\end{array}\right]=0 .
$$

The vector $\left[\begin{array}{ll}\dot{x}^{T} & \dot{y}^{T}\end{array}\right]^{T}$ is directed tangentially along the flow, so it must satisfy (13) at a border collision bifurcation. Furthermore, differentiating (8) and substituting (7) gives,

$$
\begin{aligned}
0 & =\frac{\partial g}{\partial x} \dot{x}+\frac{\partial g}{\partial y} \dot{y} \\
\Rightarrow 0 & =g_{x} f(x, y)+g_{y} v
\end{aligned}
$$

where for notational convenience $v$ replaces $\dot{y}$.

A single degree of freedom is available for varying parameters to find a bifurcation value. It follows from (6) that system parameters $\lambda$ are incorporated into the initial conditions $x_{0}$. Therefore the single degree of freedom can be achieved by parameterization $x_{0}(\theta)$, where $\theta$ is a scalar.

Grazing bifurcation points are therefore described by combining together the flow definition (9) (appropriately parameterized by $\theta$ ), algebraic equations (8), target hypersurface (12), and tangency conditions (13),(15), to give

$$
\begin{aligned}
& F_{1}\left(x_{g}, \theta, t_{g}\right):=\phi_{1}\left(x_{0}(\theta), t_{g}\right)-x_{g} \quad=0 \\
& F_{2}\left(x_{g}, y_{g}\right):=g\left(x_{g}, y_{g}\right) \\
& =0 \\
& F_{3}\left(x_{g}, y_{g}\right):=b\left(x_{g}, y_{g}\right) \\
& =0 \\
& F_{4}\left(x_{g}, y_{g}, v\right):=\left[\begin{array}{ll}
b_{x} & b_{y} \\
g_{x} & g_{y}
\end{array}\right]_{\left(x_{g}, y_{g}\right)}\left[\begin{array}{c}
f\left(x_{g}, y_{g}\right) \\
v
\end{array}\right]=0 .
\end{aligned}
$$

This set of equations may be written compactly as

$$
F_{g}\left(x_{g}, y_{g}, \theta, t_{g}, v\right)=0
$$

where $F_{g}: \mathbb{R}^{n+2 m+2} \rightarrow \mathbb{R}^{n+2 m+2}$. Solution of (20) can be achieved using Newton's method [5]. The solution process involves numerical simulation to obtain the flow (16), and is therefore classed as a shooting method.

\subsection{Limit cycles}

Periodic behaviour of limit cycles implies that the system state returns to its initial value every cycle. ${ }^{1}$ This can be expressed in terms of the flow as $x^{*}=\phi_{1}\left(x^{*}, T\right)$ where $T$ is the limit cycle period. For non-autonomous systems, the period $T$ is a known quantity. However it is not known a priori for autonomous systems. The unknown period, or return time $\tau_{r}$, can be found using Poincaré map concepts [8]. It is a function of the limit cycle starting point, so autonomous limit cycles can be expressed as

$$
x^{*}=\phi_{1}\left(x^{*}, \tau_{r}\left(x^{*}\right)\right) .
$$

Computationally this case is more interesting, so is the focus of the paper.

For a limit cycle to undergo a grazing bifurcation, (16)-(19) must be satisfied along with (21). These extra conditions can generically only be met by increasing the degrees of freedom beyond the single parameter $\theta$ of (16). In fact it becomes necessary to allow all initial conditions $x_{0}$ to vary. Accordingly, (16) becomes

$$
F_{1}^{\prime}\left(x_{g}, x_{0}, t_{g}\right):=\phi_{1}\left(x_{0}, t_{g}\right)-x_{g}=0
$$

and (21) can be expressed as

$$
F_{5}\left(x_{0}\right):=\phi_{1}\left(x_{0}, \tau_{r}\left(x_{0}\right)\right)-x_{0}=0 .
$$

Combining together (17)-(19), (22) and (23) gives

$$
F(z)=F\left(x_{g}, y_{g}, x_{0}, t_{g}, v\right)=\left[\begin{array}{c}
F_{1}^{\prime}\left(x_{g}, x_{0}, t_{g}\right) \\
F_{2}\left(x_{g}, y_{g}\right) \\
F_{3}\left(x_{g}, y_{g}\right) \\
F_{4}\left(x_{g}, y_{g}, v\right) \\
F_{5}\left(x_{0}\right)
\end{array}\right]=0
$$

where $z=\left[\begin{array}{lllll}x_{g}^{T} & y_{g}^{T} & x_{0}^{T} & t_{g} & v^{T}\end{array}\right]^{T}$ and $F: \mathbb{R}^{2 n+2 m+1} \rightarrow \mathbb{R}^{2 n+2 m+2}$

Notice though that $F$ defines an over-determined set of equations, with one more equation than variable. This can be resolved by allowing one free parameter $\lambda$. To see this, recall that if system

\footnotetext{
${ }^{1}$ Limit cycles also require that the periodic trajectory is isolated in statespace.
} 
parameters $\lambda \in \mathbb{R}^{\ell}$ are incorporated into $x$ as in (6), then (5) ensures that for the $\lambda$ component of $x, \phi_{1}\left(x_{0}, t\right)=x_{0}$ always. The associated equations in (23) are redundant and can be eliminated with no loss of information. It follows that in order to achieve an exactly determined problem, one equation should be eliminated, implying that $\lambda$ should be a scalar. If $\operatorname{dim}(\lambda)=\ell>1$, the problem becomes under-determined, resulting in a solution manifold of dimension $\ell-1$, rather than point solutions. In the remainder, it shall be assumed that $\ell=1$, and that scalar $\lambda$ is the $n$-th element of $x$. Accordingly, the last equation in $F_{5}$ is deleted from $F$ in (24).

\section{SHOOTING METHOD}

\subsection{Algorithm}

Numerical solution of (24) using Newton's method amounts to iterating on the standard update formula

$$
z^{k+1}=z^{k}-\left(D F\left(z^{k}\right)\right)^{-1} F\left(z^{k}\right)
$$

where $D F$ is the Jacobian matrix

$$
D F=\left[\begin{array}{cc}
-I_{n} & 0 \\
g_{x} & g_{y} \\
b_{x} & b_{y} \\
f^{t} b_{x x}+b_{x} f_{x}+v^{t} b_{y x} & f^{t} b_{x y}+b_{x} f_{y}+v^{t} b_{y y} \\
\hat{f}^{t} g_{x x}+g_{x} f_{x}+\hat{v}^{t} g_{y x} & \hat{f}^{t} g_{x y}+g_{x} f_{y}+\hat{v}^{t} g_{y y} \\
0 & 0
\end{array}\right.
$$

with $I_{n}$ the $(n \times n)$ identity matrix, and

$$
\begin{aligned}
& \hat{f}=\left[\begin{array}{lllll}
f & & & & \\
& f & & & \\
& & f & & \\
& & & \ddots & \\
& & & & f
\end{array}\right] \in R^{m n \times m} \\
& \hat{v}=\left[\begin{array}{lllll}
v & & & & \\
& v & & & \\
& & v & & \\
& & & \ddots & \\
& & & & v
\end{array}\right] \in R^{m^{2} \times m}
\end{aligned}
$$

$g_{x x}=\left[\begin{array}{c}\frac{\partial^{2} g_{1}}{\partial x^{2}} \\ \frac{\partial^{2} g_{2}}{\partial x^{2}} \\ \vdots \\ \frac{\partial^{2} g_{m}}{\partial x^{2}}\end{array}\right] \in R^{m n \times n}, \quad g_{y x}=\left[\begin{array}{c}\frac{\partial^{2} g_{1}}{\partial y \partial x} \\ \frac{\partial^{2} g_{2}}{\partial y \partial x} \\ \vdots \\ \frac{\partial^{2} g_{m}}{\partial y \partial x}\end{array}\right] \in R^{m^{2} \times n}$

$g_{y y}=\left[\begin{array}{c}\frac{\partial^{2} g_{1}}{\partial y^{2}} \\ \frac{\partial^{2} g_{2}}{\partial y^{2}} \\ \vdots \\ \frac{\partial^{2} g_{m}}{\partial y^{2}}\end{array}\right] \in R^{m^{2} \times m}, \quad g_{x y}=\left[\begin{array}{c}\frac{\partial^{2} g_{1}}{\partial x \partial y} \\ \frac{\partial^{2} g_{2}}{\partial x \partial y} \\ \vdots \\ \frac{\partial^{2} g_{m}}{\partial x \partial y}\end{array}\right] \in R^{m n \times m}$.

The entry $\Phi_{1}$ in (26) gives the sensitivity of the flow (9) to perturbations in initial conditions $x_{0}$,

$$
\Phi_{1}\left(x_{0}, t\right) \equiv \frac{\partial \phi_{1}}{\partial x_{0}}\left(x_{0}, t\right) .
$$

The variational equations describing the evolution of trajectory sensitivities $\Phi_{1}, \Phi_{2}$ are given in [7]. Note that these quantities are defined for non-smooth trajectories generated by hybrid systems. Furthermore, if an implicit numerical integration technique is used, these sensitivities incur minimal additional computational cost.

The submatrix $D F_{5-}$ refers to the Jacobian of $F_{5}$ in (23), with the last row removed. It is shown in [8] that $D F_{5}$ is given by

$$
D F_{5}(x)=\left(I-\frac{\left.f\right|_{\tau_{r}(x)} \sigma^{T}}{\left.\sigma^{T} f\right|_{\tau_{r}(x)}}\right) \Phi_{1}\left(x, \tau_{r}(x)\right)-I
$$

where $\sigma$ is a unit vector normal to the hyperplane $\Sigma$ defining the Poincaré map.

Care must be taken in evaluating the terms of (24) and (26) that relate to trajectory solution. The flow term $\phi_{1}\left(x_{0}^{k}, t_{g}^{k}\right)$ of (22) evaluates, via numerical integration, to the value of $x$ at time $t_{g}^{k}$ along the trajectory that has initial value $x_{0}^{k}$. The associated Jacobian entries $\Phi_{1}$ and $f$ in the first row of $D F$ should also be evaluated at time $t_{g}^{k}$ along that trajectory. Similarly, $F_{5}$ in (23) involves the flow term $\phi_{1}\left(x_{0}^{k}, \tau_{r}\left(x_{0}^{k}\right)\right)$. This evaluates to $x$

$\left.\begin{array}{cc}f & 0 \\ 0 & 0 \\ 0 & 0 \\ 0 & b_{y} \\ 0 & g_{y} \\ 0 & 0\end{array}\right]$

at the point where the trajectory, starting from $x_{0}^{k} \in \Sigma$, reencounters $\Sigma .^{2}$ The associated Jacobian entry $D F_{5-}\left(x_{0}^{k}\right)$ is also evaluated at the return point, see (27).

The matrices $g_{x x}, g_{y x}, g_{x y}$ and $g_{y y}$ are usually extremely sparse. It has been found that often the error introduced into $D F$ by ignoring them has negligible effect on convergence. However situations can arise where they do affect convergence. Efficient computation of these matrices is discussed in [5].

\section{EXAMPLE}

A model of the compass gait biped robot is discussed in detail in [9], with a summary given in [10]. The biped robot can be treated as a double pendulum. Figure 2 provides a schematic representation and identifies important parameters, including the incline angle $\gamma$. The robot configuration is described by the support angle $\theta_{s}$ and the non-support angle $\theta_{n s}$

Dynamic equations describe the evolution of the state vector $x=\left[\theta_{n s} \theta_{s} \dot{\theta}_{n s} \dot{\theta}_{s}\right]^{T} \in \mathbb{R}^{4}$ during the swing phase. An event occurs when the non-support (swinging) leg collides with the ground. This establishes the triggering condition $\theta_{n s}+\theta_{s}+2 \gamma=0$. The biped robot is therefore an example of a hybrid system, with walking motion corresponding to a periodic orbit.

To illustrate the shooting algorithm, a target hypersurface was established by $b(x, y)=x_{3}-2.5=0$ where $x_{3} \equiv \theta_{n s}$. This could be interpreted as a maximum allowable non-support leg velocity. (Perhaps the robot falls apart at higher velocities!) Walking motion that just satisfied this constraint was achieved by varying the incline angle $\gamma$. Solution progress is given in Table 4, and illustrated in Fig. 3. The figure shows the initial trajectory, and final grazing limit cycle.

\footnotetext{
${ }^{2}$ This occurs at return time $\tau_{r}\left(x_{0}^{k}\right)$, though that time is not explicitly determined.
} 


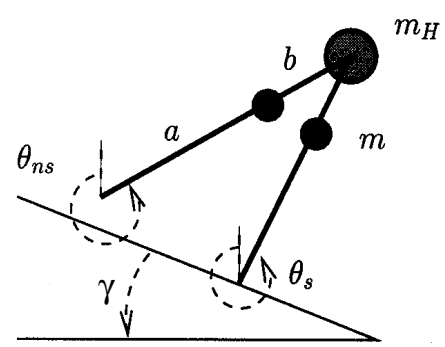

Fig. 2. Compass gait biped robot.

\begin{tabular}{|c|c|c|c|c|c|c|}
\hline \multirow{2}{*}{ Iter } & \multicolumn{3}{|c|}{ Limit cycle initial point, $x_{0}$} & \multicolumn{2}{c|}{ Grazing values } \\
\cline { 2 - 7 } & $\theta_{n s}$ & $\theta_{s}$ & $\dot{\theta}_{n s}$ & $\dot{\theta}_{s}$ & $\gamma(\mathrm{deg})$ & $t_{g}$ \\
\hline \hline 0 & -0.3500 & 0.2100 & 0.1 & -1.0000 & 3.00 & 0.2800 \\
1 & -0.4076 & 0.2253 & 0.1 & -1.1408 & 4.66 & 0.2742 \\
2 & -0.4107 & 0.2223 & 0.1 & -1.1278 & 5.07 & 0.2762 \\
3 & -0.4095 & 0.2220 & 0.1 & -1.1215 & 4.99 & 0.2764 \\
\hline
\end{tabular}

Table 1. Shooting method convergence to grazing bifurcation.

The Poincaré hyperplane $\Sigma=\left\{x: x_{3}=0.1\right\}$, with normal vector $\sigma=\left[\begin{array}{llll}0 & 0 & 1 & 0\end{array}\right]^{T}$. A projection of this hyperplane is shown in Fig. 3. The initial trajectory started from, and returned to, this hyperplane. It was not a limit cycle. The final solution started from a point on the hyperplane and returned to that same point. Along the way it grazed the surface $b(x, y)=0$.

The characteristic multipliers for the grazing limit cycle are: $-1.549,-0.1873$ and 0.0970 . Because one of these eigenvalues lies outside the unit circle, this limit cycle is non-stable. Even so, shooting method convergence was fast and reliable. On the other hand, locating this limit cycle by repeated simulations would be extremely difficult.

It can be seen from Figure 3 that this example exhibits nonsmooth (hybrid) behaviour. In this case, only one event (impact) occurs per cycle. However the shooting method is more widely applicable, to systems that undergo any (finite) number of events per cycle.

\section{CONCLUSIONS}

Hybrid systems, where discrete events have a significant influence over system behaviour, are susceptible to grazing bifurcations. This form of bifurcation refers to the situation where the system trajectory is tangential to an event triggering hypersurface. The encounter is not transversal, as required for well-defined behaviour.

Grazing bifurcation points of limit cycles can be described by a set of nonlinear, algebraic equations. Iterative solution via Newton's method requires numerical integration of the system trajectory, and therefore has the form of a shooting method. The associated Jacobian incorporates trajectory sensitivities, which can be efficiently computed along with the trajectory. The shooting method is therefore practical for arbitrarily large hybrid systems.

\section{REFERENCES}

[1] M.H. Fredriksson and A.B. Nordmark, "Bifurcations caused by grazing incidence in many degrees of freedom impact

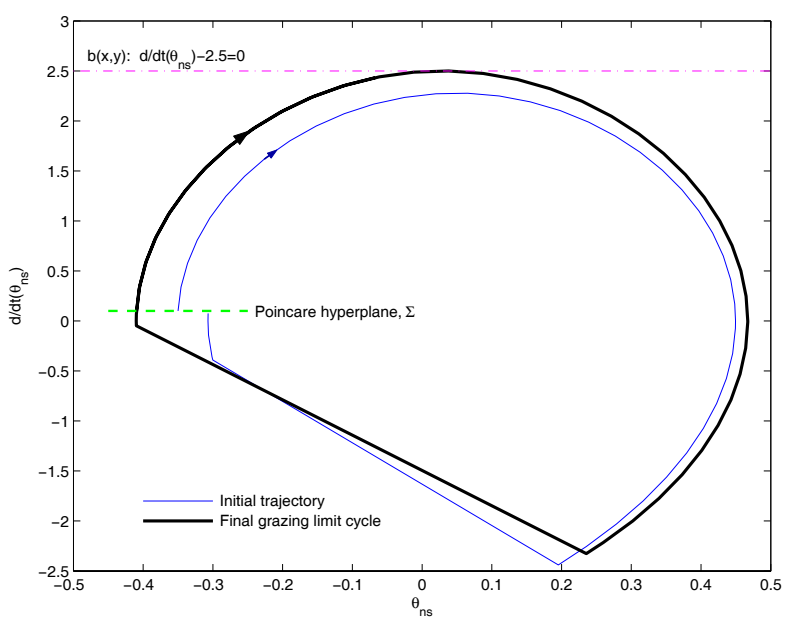

Fig. 3. Non-support leg angle $\theta_{n s}$ versus velocity $\dot{\theta}_{n s}$.

oscillators," Proceedings Royal Society London A, vol. 453, no. 1961, pp. 1261-1276, 1997.

[2] M. di Bernardo, C.J. Budd, and A.R. Champneys, "Grazing and border-collision in piecewise-smooth systems: A unified analytical framework," Physical Review Letters, vol. 86, no. 12, pp. 2553-2556, March 2001.

[3] R. Rajaraman, I. Dobson, and S. Jalali, "Nonlinear dynamics and switching time bifurcations of a thyristor controlled reactor circuit," IEEE Transactions on Circuits and Systems I, vol. 43, no. 12, pp. 1001-1006, December 1996.

[4] E.J. Doedel, A.R. Champneys, T.F. Fairgrieve, Y.A. Kuznetsov, B. Sandstede, and X.-J. Wang, "AUTO97: Continuation and bifurcation software for ordinary differential equations," Tech. Rep., Department of Computer Science, Concordia University, Montreal, Canada, March 1998, (Available from http: //cmvl.cs.concordia.ca/auto/).

[5] V. Donde and I.A. Hiskens, "Shooting for border collision bifurcations in hybrid systems," in Proceedings of the 42nd IEEE Conference on Decision and Control, Maui, HI, December 2003.

[6] I.A. Hiskens, "Power system modeling for inverse problems," IEEE Transactions on Circuits and Systems I, March 2004, To appear.

[7] I.A. Hiskens and M.A. Pai, "Trajectory sensitivity analysis of hybrid systems," IEEE Transactions on Circuits and Systems I, vol. 47, no. 2, pp. 204-220, February 2000.

[8] T.S Parker and L.O. Chua, Practical Numerical Algorithms for Chaotic Systems, Springer-Verlag, New York, NY, 1989.

[9] A. Goswami, B. Thuilot, and B. Espiau, "A study of the passive gait of a compass-like biped robot: symmetry and chaos," International Journal of Robotics Research, vol. 17, no. $15,1998$.

[10] I.A. Hiskens, "Stability of hybrid system limit cycles: Application to the compass gait biped robot," in Proceedings of the 40th IEEE Conference on Decision and Control, Orlando, FL, December 2001. 